\title{
ANALYSIS OF EFFECTIVENESS OF USING WAVELET TRANSFORMATIONS FOR REACTIVE POWER COMPENSATION
}

\author{
Igor Vorotnikov, Maxim Mastepanenko, Shaliko Gabrielyan, Anna Shunina \\ Stavropol State Agrarian University, Russia \\ mma_26@inbox.ru, aa_shu@mail.ru
}

\begin{abstract}
In recent years, there has been a significant increase in energy consumption and the algorithmization of the functioning of control devices, protection and diagnostics of electric power facilities and systems, which are based on new methods of analysis and synthesis, coming to the fore. Higher harmonics, which occur due to an increase in the share of electric receivers that have a nonlinear volt-ampere characteristic, lead to additional losses in current-carrying parts, a decrease in the service life of elements, and so on. These negative consequences reduce the energy efficiency of individual energy systems. In addition, there is a decrease in economic interest of consumers in part of reactive power compensation, which leads to a reduced energy supply reliability and efficiency of electricity in general. The mathematical apparatus of the wavelet transform is increasingly being used to analyze and describe the dynamics of complex nonlinear reactive power compensation processes. Wavelets are used as a hierarchical basis for analyzing perturbations in wide frequency ranges under conditions of non-stationary processes under study. In contrast to the Fourier transform, the wavelet transform provides a three-dimensional interpretation of the current (voltage, power) function under study, primarily in physical space (instantaneous value, time) and in frequency space (amplitude, frequency). In this article, the authors analyze the effectiveness of using wavelet transformations in the field of reactive power compensation for non-sinusoidal non-stationary modes of electric power systems.
\end{abstract}

Keywords: compensation, current, voltage, power, non-stationary loads, reactive power compensation device, energy components of instantaneous current.

\section{Introduction}

The topic of the importance of rational energy consumption is relevant all over the world. Currently, energy is a fundamental branch of the world economy, because the process of idustrialization and digitization of modern society depends on the energy component. For the development of economic activity, it is necessary that the energy industry has the following properties: reliability, effective functionality and ensuring uninterrupted supply to the consumer.

Every year the implementation of these properties becomes more difficult due to the changes in the energy supply sector, the rapid development of the energy industry, the process of transition from one energy supply system to another, more innovative types.

There are several official documents regulating all mandatory procedures for participants in the process of generating and consuming energy and issues of electricity quality in Russia.

The main documents are:

- Federal law No. 261-FZ "On energy saving and energy efficiency improvement and on amendments to certain legislative acts of the Russian Federation" [1];

- The State standard of the Russian Federation 32144-2013 “ Electric energy. Compatibility of technical means is electromagnetic standards of quality of electric energy in general-purpose power supply systems" [2].

In recent years there has been a significant increase in energy consumption. The process of algorithmization of the functioning of control devices, protection and diagnostics of electric power facilities and systems based on new methods of analysis and synthesis is becoming more relevant.

The concept of electromagnetic compatibility in terms of ensuring the quality of electricity covers all interactions between various devices and systems that use electromagnetic phenomena.

There are harmonic components of the current in modern networks. Harmonics are generated by electrical equipment with a non-linear load, which leads to malfunctions, including the equipment itself, and also negatively affects technological processes, which leads to losses.

Higher harmonics resulting from an increase in the proportion of electric receivers that have a non-linear volt-ampere characteristic lead to additional losses in current-carrying parts, a decrease in the service life of elements, and so on. 
In this article, the authors analyze the efficiency of using wavelet transformations in the field of reactive power compensation for non-sinusoidal non-stationary modes of electric power systems.

\section{Materials and methods}

Correlation functions of a random process are usually interpreted as a measure of the extent to which knowledge of the past of a random process makes it possible to predict its future.

Accordingly, in the proposed approach, the autocorrelation function of a random process of changing the active power at a prehistory interval is used to predict the active power at subsequent intervals.

In other words, by predicting the correlation function and the mathematical expectation of the current random process, you can also predict the random process itself [3; 4].

The mathematical apparatus of the wavelet transform is increasingly used to analyze and describe the dynamics of complex nonlinear processes of reactive power compensation. Wavelets are used as a hierarchical basis for the analysis of disturbances in wide frequency ranges in the conditions of nonstationary processes under study.

In contrast to the Fourier transform, the wavelet transform provides a three-dimensional interpretation of the current function under study (voltage, power), primarily in physical space (instantaneous value, time) and in frequency space (amplitude, frequency) [5-7].

When solving the issue of reactive power compensation, we keep in mind that the load is not stationary (the values of currents and voltages are random values).

Failure to take this feature into account (namely, the use of known solutions that are used in sinusoidal modes) leads to an increase in energy losses due to the growth of errors in the load power values.

For example, the well-known Frieze expression for determining the orthogonal component of the nonlinear load current (compensator current) requires that the active load power and the active network voltage remain constant over the averaging interval (network voltage period), i.e., the mode is stationary $[5 ; 8]$.

The development of a tool that allows to work with mathematical models in each specific case is of fundamental importance for practice. Such tools can be a modified algorithm for controlling the reactive power compensator, the essence of which is to determine the law using probabilistic and statistical characteristics of the load operation in real time over an observation interval that is commensurate with the period of supply network voltage [9].

For non-stationary mode, it is proposed to introduce the use of the predicted value of active power at subsequent observation intervals $P_{\text {pred }}$.

$$
P_{\text {pred }}=\sqrt{(M[P])^{2}+\sigma^{2} \cdot r(\tau)},
$$

where $\quad M[P]-$ mathematical expectation of a random process of changing the active powers; $\sigma-$ standard deviation;

$r(\tau)$ - normalized autocorrelation function.

The current voltage of the network $U$ for further consideration will be considered unchanged.

For a visual representation of the proposed algorithm functioning, mathematical modeling was performed in the MathCad environment.

During the experiment, the network voltage was set as $u(t)=100 \sin (\omega \cdot t)$, the current is generated with an interference in the form of a random variable

$$
i(t)=3 \sin (\omega \cdot t)+\frac{\operatorname{rnd}(100)-5}{10}, \omega=314 \mathrm{~s}^{-1}, t=0 \ldots 0.2 \mathrm{~s} .
$$

The current change graph is shown in Figure 1.

The active power was determined by the expression

$$
P=\frac{1}{T} \int_{0}^{T} u(t) i(t) d t .
$$




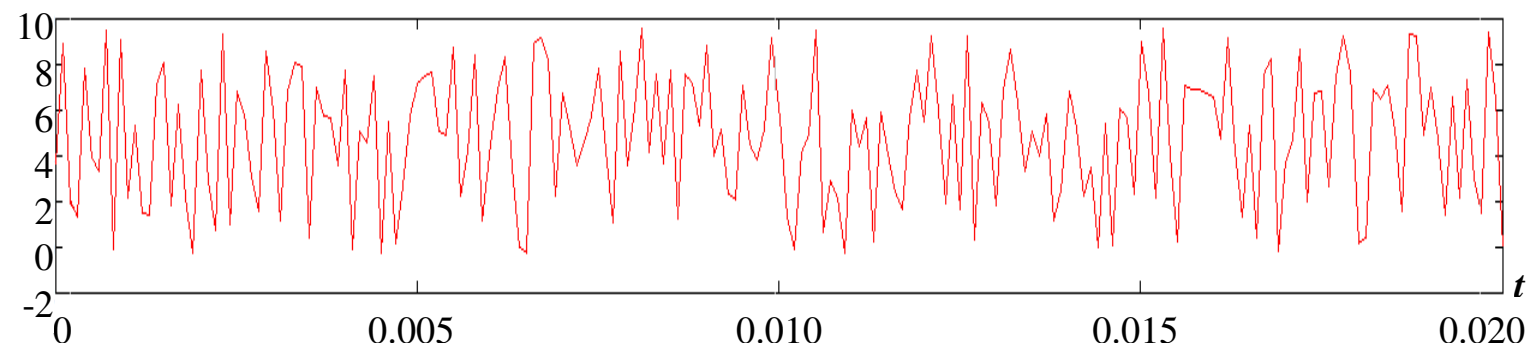

Fig. 1. Current change graph

Figure 2 shows the graph of wavelet transformations.

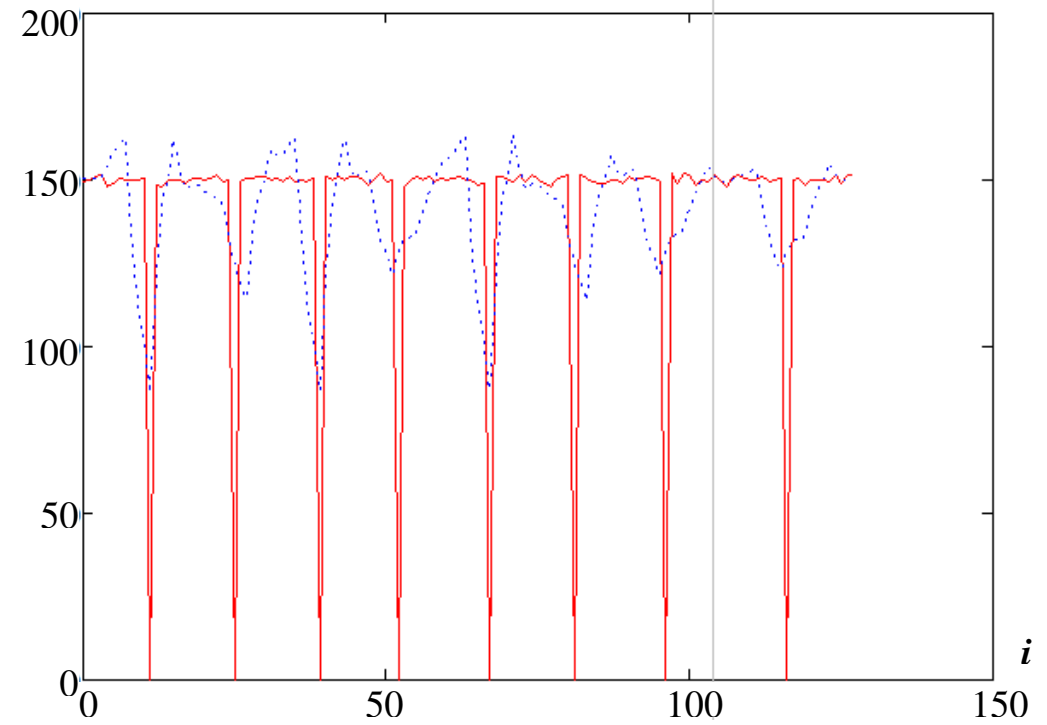

Fig. 2. Active power change graph: red $-y_{i}$, blue dashed $-s_{i}$

Table 1 shows a fragment of the final table of simulation results: random sequences of changes in active power over ten periods, mathematical expectation, standard deviation, values of autocorrelation functions of random sequences of changes in active power over ten periods with a lag of 0 and 1 , respectively, the value of the normalized autocorrelation function.

The result of calculations is the predicted value of the active power for the eleventh period $P_{\text {pred }}$.

Based on the data shown in Table 1, a mathematical simulation of the process of changing the active power was performed. The graph of active power changes is shown in Figure 3.

The red line shows the change of the power values based on finding the power values by the integrated values, the blue line shows the change of the power values, applying the proposed algorithm.

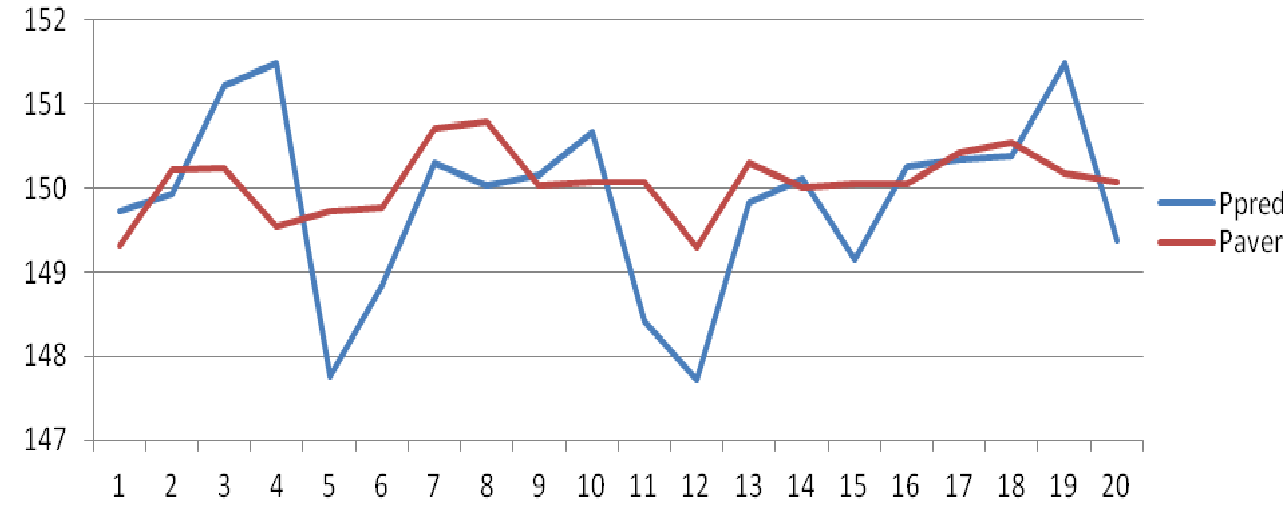

Fig. 3. Power change graph 
Simulation result

\begin{tabular}{|c|c|c|c|c|c|c|c|c|c|c|}
\hline $\begin{array}{c}\text { Period } \\
\text { No. }\end{array}$ & $\mathbf{1}$ & $\mathbf{2}$ & $\mathbf{3}$ & $\mathbf{4}$ & $\mathbf{5}$ & $\mathbf{6}$ & $\mathbf{7}$ & $\mathbf{8}$ & $\mathbf{9}$ & $\mathbf{1 0}$ \\
\hline$P_{1}, \mathrm{~W}$ & 149.71898 & 149.93014 & 151.22719 & 151.48696 & 147.76142 & 148.85234 & 150.30353 & 149.91551 & 149.73622 & 150.15278 \\
\hline$P_{2}, \mathrm{~W}$ & 150.10996 & 149.27813 & 149.71533 & 150.47401 & 150.62369 & 150.83843 & 151.2447 & 150.33375 & 146.85617 & 151.50084 \\
\hline$P_{3}, \mathrm{~W}$ & 148.54589 & 150.39918 & 152.25218 & 149.45961 & 149.8472 & 148.07884 & 150.18135 & 148.87615 & 148.88397 & 149.16816 \\
\hline$P_{4}, \mathrm{~W}$ & 149.31736 & 151.67108 & 149.18313 & 148.07925 & 150.01178 & 151.12662 & 151.48376 & 148.17195 & 150.82659 & 149.66424 \\
\hline$P_{5}, \mathrm{~W}$ & 149.19304 & 152.09279 & 151.37985 & 149.35953 & 148.48563 & 149.93929 & 149.42248 & 148.95745 & 149.08437 & 150.3378 \\
\hline$P_{6}, \mathrm{~W}$ & 148.59571 & 149.95641 & 150.0923 & 149.70105 & 151.49295 & 148.83877 & 151.54336 & 149.94368 & 151.9818 & 149.75265 \\
\hline$P_{7}, \mathrm{~W}$ & 147.94446 & 150.1502 & 151.58957 & 148.05015 & 150.52189 & 148.22514 & 149.26016 & 150.34223 & 151.18153 & 152.1758 \\
\hline$P_{8}, \mathrm{~W}$ & 149.91551 & 150.33375 & 148.87615 & 148.17195 & 148.95745 & 149.94368 & 150.34223 & 149.79836 & 150.3098 & 150.81787 \\
\hline$P_{9}, \mathrm{~W}$ & 149.73622 & 146.85617 & 148.88397 & 150.82659 & 149.08437 & 151.9818 & 151.18153 & 149.91941 & 151.20631 & 150.11305 \\
\hline$P_{10}, \mathrm{~W}$ & 150.15278 & 151.50084 & 149.16816 & 149.66424 & 150.3378 & 149.75265 & 152.1758 & 149.90673 & 148.9001 & 148.81741 \\
\hline$P_{\text {aver }}, \mathrm{W}$ & 149.32299 & 150.21687 & 150.23678 & 149.52733 & 149.71242 & 149.75776 & 150.71389 & 150.53521 & 150.18374 & 150.06328 \\
\hline$P_{\text {pred }}, \mathrm{W}$ & 149.32465 & 150.22335 & 150.24152 & 149.53149 & 149.71619 & 149.76266 & 150.71661 & 150.53605 & 150.18564 & 150.06976 \\
\hline$\sigma$ & 0.7096913 & 1.4021171 & 1.20024 & 1.123484 & 1.0691425 & 1.2201439 & 0.910469 & 0.506206 & 0.760223 & 1.401488 \\
\hline
\end{tabular}

\section{Results and discussion}

Analysis of the obtained results of mathematical modeling for 500 possible implementations of a random sequence of changes in active power shows that the variance of the predicted values (result) does not exceed the variance of the original data - a random sequence of changes in the active power at intervals of prehistory.

As shown by the analysis, methods of nonlinear mathematical programming provide the required optimization accuracy in conditions of completeness and reliability of the original information flow.

\section{Conclusions}

1. An approach to mathematical modeling of a random sequence of changes in active power is proposed, which allows to obtain predicted values of active power at any time intervals. The authors' proposed algorithm for calculating the compensator current can be used in tracking control systems of compensating installations. The proposed algorithm is valid for non-stationary modes of nonlinear loads, when using an interval of observation and control that is commensurate with the period of the supply network voltage.

2. A mathematical model of a random sequence of changes in the active power of the electric network is obtained. The main difference between this model and the existing ones is the ability to accurately describe any change in the load on various space-time hierarchies.

\section{Acknowledgements}

The research was supported by funding UDC 621.314.632.

\section{References}

[1] Federal law of 23.11.2009 N 261-FZ (ed. from 27.12.2018) "On energy saving and on improving energy efficiency and on amendments to certain legislative acts of the Russian Federation" [Electronic resource] Access from the reference.- legal system "ConsultantPlus".

[2] GOST 32144-2013 "Electric energy. Compatibility of technical means is electromagnetic. Standards of quality of electric energy in General-purpose power supply systems" [online][11.02.2020] Available at: http://docs.cntd.ru.

[3] Statistical analysis of losses of reactive energy in distribution electrical networks. Bulletin of the Savina N. V. Tsys D. A. Irkutsk state technical University. Vol. 21. No. 6 (125), 2017, pp. 79-91.

[4] Vorotnikov I., Mastepanenko M., Gabrielyan S., Shunina A. Energy estimation of parameters of reactive power compensator for non-linear loads in steady mode// Engineering for Rural Development. 2019. pp. 515-520. 
[5] Demirchyan K. S. Reactive or exchange power// Izv. USSR ACADEMY OF SCIENCES. Energy and transport. \#2, 1984, pp. 66-72.

[6] Myasoedova L. A., Myasoedov Y. V., Savina N. V. Simulation of load currents in active-adaptive distribution networks. Energy: management, quality and efficiency of energy resources use. pp. 33-40.

[7] Fryze. Active and Apparent power in non-sinusoidal systems. Przeglad Elektrot.. no 7. 1931, pp. 193-203 (In Polish).

[8] Vorotnikov I. N., Mastepanenko M. A., Gabrielyan Sh. Zh., Shunina A. A. Modified control algorithm for reactive power compensator for non-stationary loads. Vorotnikov. Electrical Engineering Russia. No. 3, 2019, pp. 11-14.

[9] System analysis of power losses in distribution power networks under conditions of uncertainty. Savina N. V.// abstract of the dissertation for the degree of doctor of technical Sciences/Institute of energy systems. L. A. melenteva of the Siberian branch of the Russian Academy of Sciences. Irkutsk. 2010. 